\title{
Sleep Quality in Chronic Obstructive Pulmonary Disease With High Risk of Acute Exacerbation
}

\author{
Chong Xing \\ First Hospital of China Medical University \\ Hui Shen \\ First Hospital of China Medical University \\ Wenyang Li \\ First Hospital of China Medical University \\ Wei Wang ( $\nabla$ wwbycmu@126.com ) \\ First Hospital of China Medical University
}

\section{Research Article}

Keywords: Chronic obstructive pulmonary disease, Overlap syndrome, Obstructive sleep apnea, Acute exacerbation, Sleep

Posted Date: April 23rd, 2021

DOI: https://doi.org/10.21203/rs.3.rs-446799/v1

License: () (7) This work is licensed under a Creative Commons Attribution 4.0 International License. Read Full License 


\section{Abstract}

This was a prospective case-control study aimed to explore the sleep quality, especially sleep-related disorders, among chronic obstructive pulmonary disease (COPD) patients with high risk of acute exacerbation, and to determine the risk factors. We enrolled COPD patients with acute exacerbation or health control visited the first hospital of China Medical University from October 1st, 2017 to October 28th, 2018. The subjective and objective sleep parameters were compared among them, and then the stepwise multiple regression analysis were performed. We found that COPD patients with high risk of acute exacerbation had decreased subjective and objective sleep quality. Patients with COPD-obstructive sleep apnea overlap syndrome revealed decreased slow wave sleep than patients with COPD alone. The subjective sleep parameters were correlated with the frequency of acute exacerbation, dyspnea index and Epworth sleepiness score. The objective sleep parameters were related to the degree of airflow obstruction, COPD Assessment Test score and the Modified British Medical Research Council dyspena score. So, we believe that the subjective and objective sleep quality of patients with high risk of acute exacerbations of COPD was poor, and the sleep quality of patients with overlap syndrome was worse than that of patients with COPD alone.

\section{Introduction}

Chronic obstructive pulmonary disease (COPD), caused by exposure to risk factors such as smoking, is characterized by chronic cough and dyspnea. The COPD patients have been up to 100 million among the over 40 years old populations in China ${ }^{1}$. Acute exacerbation is defined an acute worsening of respiratory symptoms requesting additional therapies. It can deteriorate the prognosis of COPD by declining lung function, increasing mortality and morbidity, and lowering life quality. COPD patients with history of hospitalization for exacerbation or $\geq 2$ moderate exacerbation of COPD per year are regarded as with high risk of acute exacerbation.

The influence of sleep disturbances on COPD patients is often underestimated. The previous studies mainly focused on daytime symptoms of COPD. By observing the oxygen level of COPD patients during sleep, Chaouat et al. found that the variation rate of daytime and nocturnal oxygen saturation, rather than daytime oxygen saturation, was closely related to the severity of disease and the frequency of acute exacerbation ${ }^{2}$. Dignani et al. also reported that the sleep quality of COPD patients compared with normal subjects significantly decreased ${ }^{3}$. Poor sleep could destroy immune function, induce inflammation, cause oxidative stress, and increase the prevalence of complications such as diabetes, cardiovascular disease and cognitive dysfunction. All above influences could further impair daytime function and life quality, decrease treatment compliance, cause acute exacerbation and increase mortality ${ }^{4,5}$. COPD usually combines with obstructive sleep apnea (OSA) which characterized by recurrent collapse of upper airway during sleep. It was reported that OSA could exacerbate airway inflammation and cause acute exacerbations of COPD ${ }^{6}$. The patients with OSA and COPD (known as overlap syndrome, OS) have more severe nocturnal hypoxemia and higher risk of pulmonary hypertension when compared with OSA or COPD alone. Few studies focused on the sleep status and sleep-related breathing disorders in COPD patients with high risk of acute exacerbation. This study aimed to investigate the sleep quality of COPD patients with high risk of acute exacerbation, and the influence of combining OSA on sleep.

\section{Results}

The analytical sample was composed of 39 adults, including 13 healthy control subjects, 12 COPD patients and 14 OS patients. The characteristics of them can be found in Table 1. There were 27 (69.2\%) men and 12 (30.7\%) women patients with a mean age of $64.74 \pm 8.28$ years (range, 45-83). As shown in Table 1, there was no difference among three groups except that COPD patients showed older age and higher Epworth sleepiness scale (ESS) than the control group. 
Table 1

The characteristics of the enrolled subjects

\begin{tabular}{|c|c|c|c|}
\hline & Control group $(n=13)$ & COPD group $(n=12)$ & OS group $(n=14)$ \\
\hline $\operatorname{Age}(y)$ & $59.85 \pm 6.85$ & $69.25 \pm 5.53^{\mathrm{a}}$ & $65.43 \pm 9.37^{a}$ \\
\hline Sex(male/female) & $6 / 7$ & $9 / 3$ & $12 / 2$ \\
\hline $\mathrm{BMI}\left(\mathrm{kg} / \mathrm{m}^{2}\right)$ & $24.93 \pm 3.39$ & $23.89 \pm 2.96$ & $23.22 \pm 4.66$ \\
\hline \multicolumn{4}{|l|}{ Smoking status(n) } \\
\hline current smoking & 3 & 6 & 3 \\
\hline non-smoking & 10 & 6 & 11 \\
\hline acute exacerbations(n/year) & - & $2.25 \pm 0.87$ & $1.86 \pm 1.03$ \\
\hline $\mathrm{FEV}_{1} \%$ & - & $36.97 \pm 19.38$ & $33.98 \pm 17.72$ \\
\hline $\mathrm{FEV}_{1} / \mathrm{FVC}^{2} \%$ & - & $55.49 \pm 11.68$ & $55.27 \pm 17.81$ \\
\hline FEF25-75\% & - & $18.17 \pm 9.21$ & $21.83 \pm 15.48$ \\
\hline PEF\% & - & $34.26 \pm 19.47$ & $39.06 \pm 23.59$ \\
\hline CAT score & - & $23.33 \pm 8.5$ & $24.00 \pm 8.58$ \\
\hline mMRC score & - & $2.83 \pm 1.03$ & $2.36 \pm 1.22$ \\
\hline ESS score & $6.92 \pm 4.63$ & $14.17 \pm 6.24^{\mathrm{a}}$ & $9.86 \pm 5.33$ \\
\hline \multicolumn{4}{|l|}{ Complications (n) } \\
\hline coronary artery disease & 2 & 5 & 3 \\
\hline hypertension & 6 & 4 & 4 \\
\hline \multicolumn{4}{|c|}{ Data were presented as mean $\pm S D$, a: $p<0.05$ when compared with control group } \\
\hline $\begin{array}{l}\text { BMI, body mass index; CAT, } \\
\text { sleepiness scale; mMRC, mo }\end{array}$ & Imonary disease as & D: chronic obstruct & ease; ESS, epworth \\
\hline
\end{tabular}

As expected, Pittsburgh Sleep Quality Index (PSQI) scores significantly differentiated among three groups. Compared with control group, COPD and OS group had higher scores $(\mathrm{P}<0.05)$ (Fig. 1). The objective sleep quality results showed that the COPD patients had shorter total sleep time (TST), lower sleep efficiency, higher percent of sleep time with oxygen saturation below 90\% (SIT90), more arousal and wakefulness after sleep onset (WASO) when compared with control group after adjusting for age. In addition, the slow wave sleep in COPD patients was further decreased when combined with OSA (Table 2). Table 3 shows the results of correlative analysis of sleep quality in all patients with COPD. Acute exacerbation and mMRC were negatively related to PSQI Score. WASO positively correlated to body mass index (BMI), COPD Assessment Test score and the Modified British Medical Research Council (mMRC) dyspena score. For sleep architecture, age, PEF\% and FEF25-75\% mainly influenced rapid eye movement (REM) sleep, while sex and apnea-hyponea index (AHI) affected slow wave sleep.

Multiple regression analysis was used to find the independent variables determining sleep parameters in COPD patients. Taking into account of co-founding factors and co-linearity, the models showed that acute exacerbation explained 19\% (adjusted $\mathrm{R}^{2}$ ) of the PSQI Score, mMRC score and BMI explained 33.7\% (adjusted $\mathrm{R}^{2}$ ) of the WASO, and FEF25-75\% explained 17\% (adjusted $\mathrm{R}^{2}$ ) of REM\% (Table 4). 
Table 2

The polysomnography results of the enrolled subjects

\begin{tabular}{|c|c|c|c|}
\hline Sleep parameters & Control group & COPD group & OS group \\
\hline Total sleep time(min) & $464.10 \pm 79.28$ & $350.21 \pm 91.45^{a}$ & $344.14 \pm 80.18^{a}$ \\
\hline Sleep efficiency (\%) & $88.87 \pm 7.13$ & $67.93 \pm 12.68^{a}$ & $68.11 \pm 14.83^{a}$ \\
\hline Sleep latency (min) & $21.48 \pm 30.17$ & $40.13 \pm 32.07$ & $49.18 \pm 36.58$ \\
\hline Times of arousals(n) & $6.46 \pm 6.80$ & $24.50 \pm 12.49^{a}$ & $29 \pm 10.41^{\mathrm{a}}$ \\
\hline Wake-up time after sleep onset(min) & $34.02 \pm 36.00$ & $111.54 \pm 89.18^{a}$ & $98.21 \pm 60.05^{a}$ \\
\hline Rapid eye movement sleep (\%) & $25.29 \pm 13.01$ & $20.83 \pm 14.15$ & $26.55 \pm 17.97$ \\
\hline Slow wave sleep(\%) & $24.49 \pm 8.80$ & $25.63 \pm 11.85$ & $8.59 \pm 8.71^{a b}$ \\
\hline Apnea hyponea index (h) & $3.02 \pm 1.12$ & $2.78 \pm 1.71$ & $14.47 \pm 15.35^{\mathrm{ab}}$ \\
\hline $\mathrm{MmSaO}_{2}(\%)$ & $87.00 \pm 6.65$ & $81.50 \pm 12.18$ & $80.57 \pm 7.84$ \\
\hline $\mathrm{MSaO}_{2}(\%)$ & $95.74 \pm 1.73$ & $91.92 \pm 4.78$ & $92.21 \pm 3.75$ \\
\hline $\mathrm{SIT}_{90}(\%)$ & $0.62(0.07,38.43)$ & $28.22 \pm 38.54^{a}$ & $24.14 \pm 33.69$ \\
\hline
\end{tabular}

Table 3

Correlative analysis of sleep quality in all patients with COPD

\begin{tabular}{|c|c|c|c|c|c|c|c|c|c|c|c|c|c|c|}
\hline & Age & Sex & BMI & $\mathrm{AE}$ & $\mathrm{FEV}_{1} \%$ & $\begin{array}{l}\mathrm{FEF}_{25} \\
-75 \%\end{array}$ & PEF\% & CAT & mMRC & ESS & $\mathrm{AHI}$ & $\mathrm{MSaO}_{2}$ & $\mathrm{MmSaO}_{2}$ & $\mathrm{SIT}_{90}$ \\
\hline PSQI & 0.028 & 0.011 & -0.095 & $0.471^{a}$ & -0.186 & -0.386 & -0.281 & 0.271 & $0.463^{\mathrm{a}}$ & $0.576^{a}$ & -0.128 & 0.282 & 0.270 & -0.240 \\
\hline $\begin{array}{l}\text { Total } \\
\text { sleep } \\
\text { time }\end{array}$ & -0.006 & 0.027 & 0.203 & -0.227 & 0.125 & 0.095 & -0.014 & -0.210 & -0.180 & -0.050 & 0.031 & 0.145 & -0.014 & -0.153 \\
\hline $\begin{array}{l}\text { Sleep } \\
\text { efficiency }\end{array}$ & 0.075 & 0.233 & -0.261 & -0.172 & -0.017 & -0.122 & -0.016 & -0.364 & -0.367 & -0.069 & -0.074 & -0.121 & -0.247 & 0.013 \\
\hline $\begin{array}{l}\text { Sleep } \\
\text { latency }\end{array}$ & -0.294 & -0.087 & 0.037 & 0.036 & 0.144 & 0.219 & 0.107 & 0.308 & 0.246 & -0.227 & 0.227 & 0.283 & 0.213 & -0.214 \\
\hline $\begin{array}{l}\text { Times of } \\
\text { arousals }\end{array}$ & 0.291 & -0.241 & 0.238 & -0.157 & 0.239 & 0.404 & 0.301 & -0.149 & -0.289 & -0.428 & 0.302 & 0.389 & 0.328 & -0.490 \\
\hline WASO & 0.093 & -0.222 & $0.497^{a}$ & 0.279 & -0.065 & 0.021 & $\overline{0} .091$ & $0.420^{\mathrm{a}}$ & $0.448^{a}$ & 0.388 & -0.042 & -0.069 & 0.147 & 0.083 \\
\hline REM\% & $-0.433^{a}$ & -0.233 & 0.201 & -0.066 & 0.391 & $0.406^{\mathrm{a}}$ & $0.569^{a}$ & -0.095 & -0.200 & -0.381 & 0.074 & 0.071 & 0.109 & -0.122 \\
\hline SWS\% & -0.038 & $0.397^{a}$ & -0.157 & 0.151 & -0.086 & -0.135 & -0.261 & -0.014 & 0.195 & 0.283 & $-0.516^{a}$ & 0.097 & 0.305 & -0.332 \\
\hline
\end{tabular}

Data were presented as correlation coefficients, a: $p<0.05$.

AE: acute exacerbation; AHI: apnea hyponea index; BMI, body mass index; CAT, chronic obstructive pulmonary disease assessment test; COPD: chronic obstructive pulmonary disease; ESS, epworth sleepiness scale; $\mathrm{MmSaO2}$, the nadir of nocturnal oxygen saturation; $\mathrm{MSaO2}$, the mean nocturnal oxygen saturation; mMRC, modified medical research council; PSQI: pittsburgh sleep quality index; REM: rapid eye movement sleep; SIT90, the percent of sleep time with oxygen saturation less than $90 \%$; SWS: slow wave sleep; WASO, wake after sleep onset 
Table 4

Stepwise multiple regression analysis of sleep quality in 26 COPD patients

\begin{tabular}{|c|c|c|c|c|c|}
\hline Outcome/ predictors Coefficient & Coefficient & SE & $P$ value & Partial R & Adjusted $\mathrm{R}^{2}$ \\
\hline \multicolumn{6}{|l|}{ PSQI } \\
\hline Constant & 5.66 & 1.80 & 0.004 & & \\
\hline $\mathrm{AE}$ & 2.09 & 0.80 & 0.015 & 0.22 & 0.19 \\
\hline \multicolumn{6}{|l|}{ WASO } \\
\hline Constant & -154.15 & 75.01 & 0.051 & & \\
\hline BMI & 8.28 & 3.11 & 0.014 & & \\
\hline mMRC & 24.69 & 10.67 & 0.030 & 0.39 & 0.34 \\
\hline \multicolumn{6}{|l|}{ REM\% } \\
\hline Constant & 13.93 & 4.97 & 0.010 & & \\
\hline $\mathrm{FEF}_{25-75 \%}$ & 15.67 & 6.34 & 0.021 & 0.20 & 0.17 \\
\hline
\end{tabular}

\section{Discussion}

COPD patients were usually observed with decreased sleep quality and sleep-related breathing disorders. The study of Ding et al. demonstrated that, in addition to the treatment of daytime symptoms, it was necessary to solve sleep disorders in COPD patients to improve their life quality ${ }^{7}$. However, Sanders et al. analyzed polysomnography data in 1138 community-based COPD patients with mild airflow obstruction, and noted that there were only minor differences in sleep quality and structure between these patients and controls ${ }^{8}$. Up to now, the guidelines of COPD management did not give much advice about it. Scharf et al. investigated PSQI scale of 1117 patients with moderate and severe COPD at 3 months and 12 months after admission respectively ${ }^{9}$. They found these patients were more likely to suffer from decreased sleep quality, and this change remained for 12 months in half of the patients. However, little data focused on sleep disorders in COPD patients with high risk of acute exacerbations. Our study showed that compared with the controls, COPD patients with high risk of acute exacerbation showed not only poorer sleep quality manifested as shorter TST, lower sleep efficiency and more arousals and wakefulness, but also more risk to combine with OSA. When COPD and OSA coexisted, sleep quality of the patients further decreased and sleep structure was damaged as reduced slow wave sleep. Further analysis showed that acute exacerbation, high mMRC and FEF25-75\% more closely related to sleep disorders.

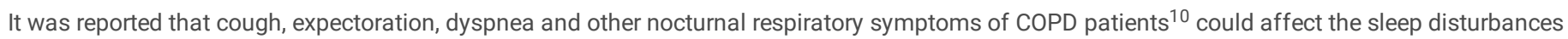
including the difficulties in initiating and maintaining sleep and the increasing numbers of arousals during the night. As we all know, COPD patients with high risk of acute exacerbation usually have more symptoms and lower oxygen level because of prominent airway and systemic inflammation, increased sympathetic nerve activity and increase oxidative stress ${ }^{11}$. Our study discovered that acute exacerbation was positively related to PSQI, and higher mMRC score meant longer wakeful time during night. The increased inspiratory load due to hyperinflation in COPD patients with high risk of acute exacerbation could add the work of breathing and elicit arousals by stimulation of mechanoreceptors in the chest wall and lower airways ${ }^{12}$. Besides, it could also lead to autonomic nervous dysfunction ${ }^{13}$ and have an adverse effect on sleep. A Canadian study who investigated PSQI Scale of 574 COPD patients and followed up for 18 months showed that poor sleep quality of COPD patients was linked to increased risk of exacerbations ${ }^{14}$.

In addition, chronic sleep deprivation could elevate the levels of inflammatory markers and caused immune deficiency ${ }^{15}$. The study of Prather et al. reported that adults who slept less than five hours a night or had sleep disturbances were more likely to suffer from cold and infection such as influenza and pneumonia ${ }^{16}$, which might explain that COPD patients with decreased sleep quality were more prone to acute exacerbation. Ajili et al. found that the average number of acute exacerbations per year in COPD patients with sleep disorders was greater than that in patients without sleep disorders ${ }^{17}$. It may be the cause that sleep disorders could increase the degree of airflow obstruction and then result in an acute exacerbation of COPD ${ }^{18}$. These results indicated that the decline of sleep quality and acute aggravation of COPD could promote each other in a form of vicious circle.

The overlap of COPD and OSA was not common in the general population (1.0-3.6\%). For COPD patients, the prevalence of coexisted OSA varied from 2.9$65.9 \%{ }^{19}$. Our study further found COPD patients with high risk of acute exacerbations had higher risk of sleep-related breathing disorders. Possible reasons for a higher prevalence of OSA among COPD patients with high risk of acute exacerbations include the increase of using inhaled or systemic steroid use or upper respiratory tract stenosis caused by the transfer of edema fluid from the lower extremities to the neck in those with cor-pulmonale and upper airway myopathy due to either COPD itself ${ }^{20}$. It may also be related to changes in sleep-related behaviors associated with worsening disease when COPD patients with high risk of acute exacerbations. In overlap condition, the decreased sleep quality was more noticeable and sleep structure was further damaged when compared with COPD alone. OSA itself usually induces nocturnal hypoxia and sleep fragment, so the overlap of COPD and OSA could bring lower oxygen saturation, more severe oxidant stress and poorer sleep quality. We also found the changed sleep structure manifested by the decreased slow wave sleep in overlap condition. Hill et al. found that sleep was involved in antioxidant processes through a new study of short sleep Drosophila melanogaster mutants ${ }^{21}$. Sleep loss may make individuals more sensitive to oxidative stress and related diseases. On the contrary, pathological destruction of antioxidant response may also lead to sleeping loss as well as pathological changes in related diseases. Besides, Kohli et al. found that alterations in the sleep-wake cycle were associated with 
elevations in inflammatory markers ${ }^{22}$. It has been demonstrated that patients with OSA have increased chronic inflammation for the sleep fragmentation and chronic intermittent hypoxia as well as impaired gas exchange similarly facilitates chronic inflammation in patients with COPD ${ }^{20}$. Therefore, attention should be given to the presence of OSA for COPD patients with particularly poor sleep quality.

At present, there is controversy about the relationship between sleep disorders and FEV1\% or FVC\% in patients with COPD. Krachman et al. studied the sleep quality of 25 patients with severe COPD and found that FEV1\% and FVC\% was associated with sleep quality ${ }^{23}$. However, Donovan and coworkers suggested that sleep disorders in COPD patients were independent of airflow limitation and had nothing to do with airflow blockage ${ }^{24}$. Our data showed that FEF25-75\% and PEF\% were positively correlation with REM\%. It may be caused by peripheral airway blockage and decreased peak flow velocity in patients with COPD. It leaded to shortness of breath, activation of the reticular activation system, release of catecholamine and then a decreased slow wave sleep in COPD patients ${ }^{25}$. Our analysis showed that sleep quality was not linked to FEVI\% and FVC\%. The results were consistent with what Roberts and Cleutjens observed about the relationship between sleep quality and lung function parameters ${ }^{26,27}$. Roberts et al. studied the sleep quality of 337 patients with COPD and found that no correlations were seen between sleep quality and spirometry values as measured by FEV $1 \%$ or VC\% ${ }^{26}$. Similarly, Cleutjens et al. found that sleep disturbances did not differ across GOLD grades classified by FEV1/FVC among 562 ambulatory patients with COPD 27.

In summary, we have found that COPD patients with high risk of acute exacerbation had decreased sleep quality when assessed by subjective and objective scores. The sleep structure was further damaged when combined with OSA. These suggested that clinicians should pay much attention to sleep evaluation for these patients. The abnormal sleep and sleep-related breathing disorders should be identified early and given appropriate intervention to improve life quality and prognosis of COPD patients with high risk of acute exacerbation.

\section{Methods}

Subjects. From October 1, 2017 to October 28, 2018, 26 stable COPD patients ( 21 males and 5 females, aged $45-77$ years) with high risk of acute exacerbation and 13 normal controls (control group) were enrolled from the Department of Respiratory and Critical Care Medicine of the First Hospital of China Medical University. Inclusion criteria: 1) COPD was diagnosed following with GOLD 2018, and COPD patients who had history of hospitalization for exacerbation or $\geq 2$ moderate exacerbation of COPD per year were regarded as with high risk of acute exacerbation. 2) OSA was diagnosed in accordance with the updated 2014 American Academy of Sleep Medicine (AASM) criteria ${ }^{28}$. Exclusion criteria: 1) no more than 40 years old. 2) the subjects with other diseases that affected the airflow and the quality of sleep, such as narcolepsy, mental illness, severe myocardial infarction, arrhythmia, severe liver and kidney insufficiency. 3) the subjects who could not complete the questionnaires because of cognitive impairment. 4) the subjects who were taking drugs that affected sleep quality. Based on the results of overnight polysomnography, the COPD patients were divided into COPD group $(n=12)$ and OS group $(n=14)$. This study was approved by the Ethics Committee of the First Hospital of China Medical University, and all subjects signed informed consents. All procedures performed in studies involving human participants were in accordance with the ethical standards of the institutional and/or national research committee and with the 1964 Helsinki declaration and its later amendments or comparable ethical standards.

Questionnaires. CAT score (GlaxoSmithKline, Brent ford, UK) ${ }^{29}$ were recorded to evaluate the daily symptoms of the subjects. The severity of dyspnea was quantified by the mMRC dyspnea scale ${ }^{30}$. The PSQI ${ }^{31}$ was used to subjectively evaluate sleep quality which had 19 items and was divided into 7 subparts with a range of 0-21 and a score above 5 indicated poor sleep. The ESS ${ }^{32}$ was employed to classify subjective daytime sleepiness which contained eight items ranging from 0-24 and a high score indicated excessive daytime sleepiness.

Pulmonary function test. When they came into stable stage, all COPD patients had pulmonary function testing (Master Screen, Germany) based on American Thoracic Society standards ${ }^{33}$. Before the test, the safety and accuracy of implementation was evaluated. The subjects were required to meet inclusion criteria and take no bronchodilators within 2 weeks. Pre-bronchodilator spirometry was performed according to American Thoracic Society Standards and repeated 15 minutes after inhalation of $400 \mu \mathrm{g}$ salbutamol via large-volume spacer. At least three measurements were taken and the best value was selected for analysis. FEV1\%, FVC\%, FEV1/FVC\%, FEF25-75\%, PEF and PEF\% were recorded. Airflow limitation was diagnosed when the ratio of FEV1 to FVC was less than 70\% predicted after bronchodilator inhalation.

Polysomnography. All subjects underwent a full overnight PSG (Respironics, Alice 5, US) to assess objective sleep quality just after pulmonary function testing in the same day by monitoring and recording all night electroencephalography (EEG), electrocardiography (ECG), electrooculography (EOG), chin and tibial electromyography (EMG), respiration, ribcage and abdominal movements, snoring, body position and oxygen saturation by finger pulse oximetry. Apneahypopnea index $(\mathrm{AHI})$ was calculated as the sum of apneas and hypopneas during the sleep period divided by total sleep time. Apnea was defined as a cessation of air flow for more than 10 seconds and hypopnea as a reduction of air flow $>50 \%$ for $>10$ seconds plus oxygen desaturation of $>3 \%$ or arousal. Arousals meant an abrupt shift of EEG frequency lasting at least 3 seconds, with at least 10 seconds of preceding stable sleep. TST, sleep latency, WASO, sleep architecture, $\mathrm{AHI}$, the nadir of nocturnal oxygen saturation (MmSaO2), the mean nocturnal oxygen saturation (MSaO2), SIT90 were recorded and compared. The data analyzers were blinded to the patient's clinical condition.

Statistical Analysis. The data were statistically analyzed by SPSS (Statistical Product and Service Solutions 20.0 version, Armonk, NY, USA) software. Descriptive and inferential statistics were used to characterize baseline measurements. Normally distributed data were expressed as mean \pm standard deviation (SD), median and interquartile range, or number. Comparisons of mean levels of quantitative variables between groups were assessed by using the one-way ANOVA or in-dependent t-tests or Mann-Whitney $U$ test. Proportions were compared between groups by using Fisher exact chi-square testing. Covariance analysis or multiple linear regression correction was used to correct confounding factors such as age. Univariate associations between sleep parameters and clinical variables were performed with Pearson (variables with equidistant and normal distribution), Spearman (variables that do not conform 
to normal distribution) or Kendall's tau-b correlation coefficients (the classified variables). Stepwise multiple regression was used to identify which variables could predict the sleep quality. $P<0.05$ were considered statistically significant.

\section{Declarations}

\section{Data Availability}

Te datasets generated during and/or analyzed during the current study are available by request.

\section{Acknowledgements}

This work was supported by grants from the National Key Research and Development Program of China (No.2016YFC1304502) and the Science and Technology Planning Project of Liaoning Province, China (No.2017225022).

\section{Author contributions}

XC: study design, data collection, statistical analysis, and initial manuscript drafting.SH: data analysis and critical revision of the manuscript. L-WY: participant selection and data collection. WW: study design, result interpretation, critical revision and final approval of the manuscript.

\section{Competing interests}

The author reports no competing interests.

\section{References}

1. Wang C, et al. Prevalence and risk factors of chronic obstructive pulmonary disease in China (the China Pulmonary Health [CPH] study): a national crosssectional study. The Lancet. 391 (10131):1706-1717 (2018).

2. Chaouat A, et al. Outcome of COPD patients with mild daytime hypoxaemia with or without sleep-related oxygen desaturation. European Respiratory Journal. 17 (5):848-855 (2001).

3. Dignani L, Toccaceli A, Lucertini C, Petrucci C, Lancia L. Sleep and quality of life in people with COPD: a descriptive-correlational study. Clinical nursing research. 25 (4):432-447 (2016).

4. Agusti A, Hedner J, Marin JM, Barbé F, Cazzola M, Rennard S. Night-time symptoms: a forgotten dimension of COPD. Eur Respir Rev. 20(121):183-94 (2011).

5. Omachi TA, Blanc PD, Claman DM, Chen H. Disturbed sleep among COPD patients is longitudinally associated with mortality and adverse COPD outcomes. Sleep Medicine. 13 (5):476-483 (2012).

6. Wang Y, et al. Obstructive sleep apnea exacerbates airway inflammation in patients with chronic obstructive pulmonary disease. Sleep Medicine. 16 (9):1123-1130 (2015).

7. Ding B, Small M, Bergström G, Holmgren U. A cross-sectional survey of night-time symptoms and impact of sleep disturbance on symptoms and health status in patients with COPD. Int J Chron Obstruct Pulmon Dis. 12:589-599 (2017).

8. Sanders MH, et al. Sleep and Sleep-disordered Breathing in Adults with Predominantly Mild Obstructive Airway Disease. Am J Respir Crit Care Med. 167 (1):7-14 (2003).

9. Scharf SM, Krachman SL, McEvoy C, Harnden S, Criner GJ. Sleep Quality In Patients With Moderate To Severe COPD. A5816. https://doi.org/10.1164/ajrccm-conference.(2016)

10. Miravitlles M, Ribera A. Understanding the impact of symptoms on the burden of COPD. Respir Res. 18(1):67(2017).

11. Wu KL, Chan SH, Chan JY. Neuroinflammation and oxidative stress in rostral ventrolateral medulla contribute to neurogenic hypertension induced by systemic inflammation. J Neuroinflammation. 9:212 (2012).

12. Eckert DJ, Younes MK. Arousal from sleep: implications for obstructive sleep apnea pathogenesis and treatment. J Appl Physiol (1985). 116(3):302-313 (2014).

13. O'Donnell DE, Webb KA, Neder JA. Lung hyperinflation in COPD: applying physiology to clinical practice. COPD Res\&Prac. 1 (1):4(2015).

14. Shorofsky M, et al. A Population Based Study of Sleep Disorders in Chronic Obstructive Pulmonary Disease: Sleep Quality and Acute Exacerbations of COPD. American Thoracic Society, A5995. https://doi.org/10.1164/ajrccm-conference (2016).

15. Besedovsky L, Lange T, Born J. Sleep and immune function. Pflugers Arch. 463(1):121-137 (2012).

16. Prather AA, Leung CW. Association of Insufficient Sleep With Respiratory Infection Among Adults in the United States. JAMA Intern Med. 176(6):850-852 (2016).

17. Ajili IME, Moatemri Z, Daboussi S, Mhamdi S, Aichaouia C, Cheikh R. Sleep quality, fatigue and mental health status among mild to severe COPD patients. Eur Respiratory Soc. https://doi.org/10.1183/13993003.congress-2016.PA3738 (2016).

18. İn E, Turgut T, Özdemir C. Impact of Restless Legs Syndrome on the Sleep Quality in Chronic Obstructive Pulmonary Disease. Eurasian J Pul. 18 (1):18-23 (2016).

19. Shawon MSR, Perret JL, Senaratna CV, Lodge C, Hamilton GS, Dharmage SC. Current evidence on prevalence and clinical outcomes of co-morbid obstructive sleep apnea and chronic obstructive pulmonary disease: A systematic review. Sleep Med Rev. 32:58-68(2017). 
20. Malhotra A, et al. Research Priorities in Pathophysiology for Sleep-disordered Breathing in Patients with Chronic Obstructive Pulmonary Disease. An Official American Thoracic Society Research Statement. Am J Respir Crit Care Med. 197 (3):289-299 (2018).

21. Hill VM, et al. A bidirectional relationship between sleep and oxidative stress inDrosophila. Plos Biology. 16 (7):e2005206 (2018).

22. Kohli P, et al. Functional Capacity, Health Status and Inflammatory Biomarker Profile in a Cohort of Patients with COPD. J Cardiopulm Rehabil Prev. 35(5):348 (2015).

23. Krachman SL, et al. Physiologic correlates of sleep quality in severe emphysema. COPD. 8(3):182-188 (2011).

24. Donovan LM, et al. Airflow Obstruction Is Associated With Lower Ratings Of Sleep Disturbance In Patients With Clinically Identified COPD. A4833. https://doi.org/10.1164/ajrccm-conference (2017).

25. Bratel T, Wennlund A, Carlström K. Impact of hypoxaemia on neuroendocrine function and catecholamine secretion in chronic obstructive pulmonary disease (COPD). Effects of long-term oxygen treatment. Respir Med. 94(12):1221-1228 (2000).

26. Roberts MM, Cho J-G, Wheatley JR. The Prevalence Of Poor Sleep Quality In Patients With Chronic Obstructive Pulmonary Disease Referred For Pulmonary Rehabilitation. American Thoracic Society. A3567. https://doi.org/10.1164/ajrccm-conference (2016).

27. Cleutjens FA, Pedone C, Janssen DJ, Wouters EF, Incalzi RA. Sleep quality disturbances and cognitive functioning in elderly patients with COPD. ERJ open research. 2(3):00054-02016 (2016).

28. Berry RB, et al. AASM scoring manual updates for 2017 (version 2.4). American Academy of Sleep Medicine. https://doi.org/10.5664/jcsm.6576 (2017)

29. Stanford RH, et al. Assessment of the COPD Assessment Test Within U.S. Primary Care. Chronic Obstr Pulm Dis. 7(1):26-37 (2020).

30. Stenton C. The MRC breathlessness scale. Occup Med. 58(3):226-227 (2008).

31. Culver BH, et al. Recommendations for a Standardized Pulmonary Function Report. An Official American Thoracic Society Technical Statement. Am J Respir Crit Care Med. 196 (11):1463-1472 (2017).

32. Mollayeva T, Thurairajah P, Burton K, Mollayeva S, Shapiro CM, Colantonio A. The Pittsburgh sleep quality index as a screening tool for sleep dysfunction in clinical and non-clinical samples: A systematic review and meta-analysis. Sleep Med Rev. 25:52-73(2016).

33. Chiu HY, et al. Diagnostic accuracy of the Berlin questionnaire, STOP-BANG, STOP, and Epworth sleepiness scale in detecting obstructive sleep apnea: a bivariate meta-analysis. Sleep Med Rev. 36:57-70(2017).

\section{Figures}




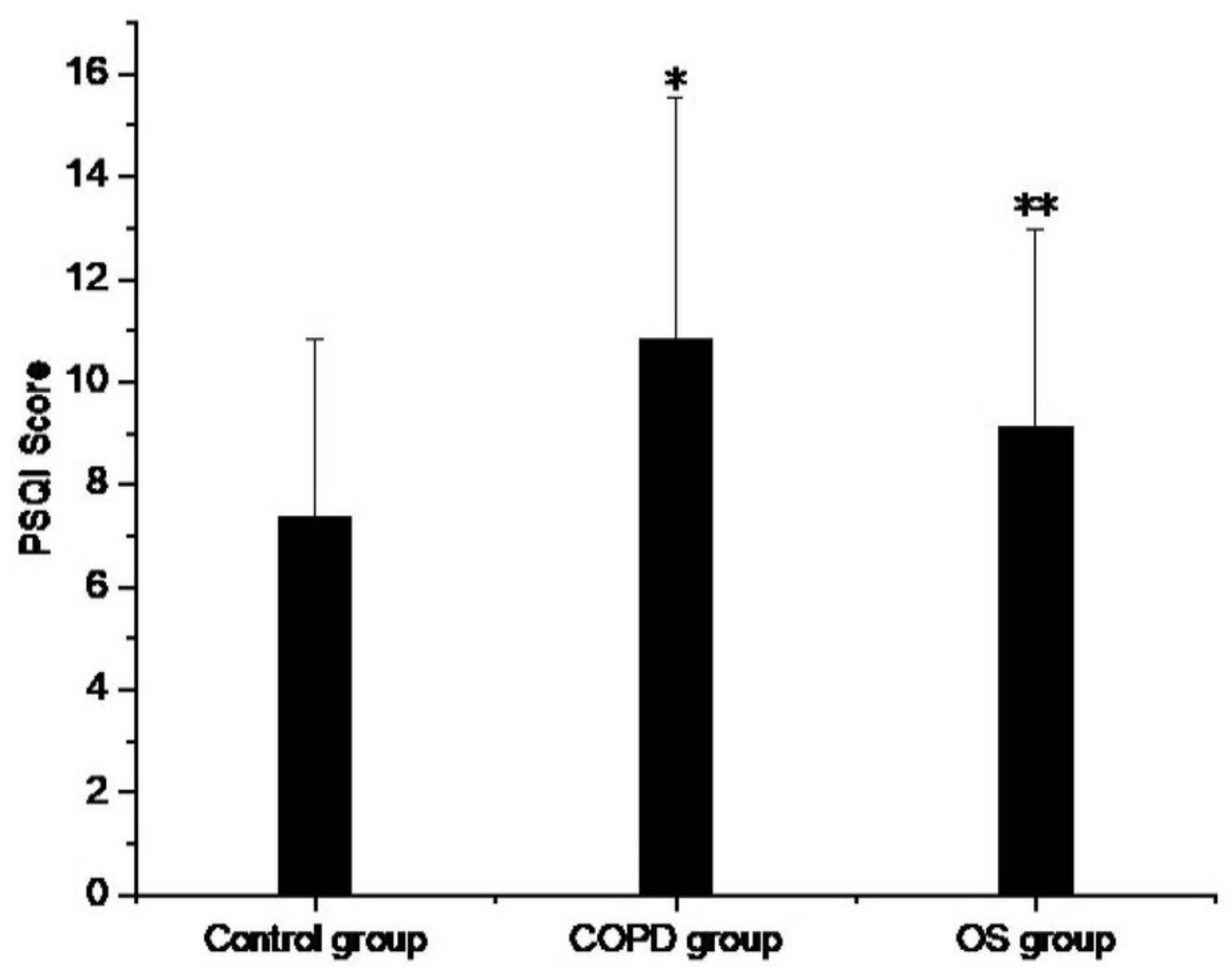

Figure 1

The comparison of PSQI score among three groups * $p<0.05$ when compared with control group, ** $p<0.05$ when compared with COPD group COPD: chronic obstructive pulmonary disease; OS, overlap syndrome; PSQI: pittsburgh sleep quality index 